This document is confidential and is proprietary to the American Chemical Society and its authors. Do not copy or disclose without written permission. If you have received this item in error, notify the sender and delete all copies.

\title{
Interaction of water with graphene/Ir(111) studied by vibrational spectroscopy
}

\begin{tabular}{|r|l|}
\hline Journal: & Langmuir \\
\hline Manuscript ID & Draft \\
\hline Manuscript Type: & Article \\
\hline Date Submitted by the & $\mathrm{n} / \mathrm{a}$ \\
\hline Complete List of Authors: & $\begin{array}{l}\text { Glei\&szlig;ner, Robert; Deutsches Elektronen-Synchrotron, FS-NL } \\
\text { Creutzburg, Marcus; Deutsches Elektronen-Synchrotron, DESY NanoLab } \\
\text { Noei, Heshmat; Deutsches Elektronen-Synchrotron, Research Group X- } \\
\text { ray Physics and Nanoscience } \\
\text { Stierle, Andreas; Deutsches Elektronen-Synchrotron, FS-NL }\end{array}$ \\
\hline
\end{tabular}

\section{SCHOLARONE \\ Manuscripts}




\title{
Vibrational fingerprint of graphene supported
} frozen water clusters

\author{
Robert Gleißner, ${ }^{\dagger, \dagger}$ Marcus Creutzburg, ${ }^{\dagger, \dagger}$ Heshmat Noei, ${ }^{* \dagger}$ and Andreas Stierle ${ }^{\dagger, \ddagger}$ \\ †Deutsches Elektronen-Synchrotron (DESY), Hamburg, Germany \\ $\ddagger$ Fachbereich Physik, Universität Hamburg, Hamburg Germany \\ E-mail: heshmat.noei@desy.de \\ Phone: +49 (0)40 89986011
}

\begin{abstract}
Water in confinement exhibits altered properties in molecular arrangement, bonding and interaction with its neighboring environment, as compared to its bulk counterpart. In this work, periodically arranged $\mathrm{D}_{2} \mathrm{O}$ nano droplets of $\sim 1 \mathrm{~nm}$ size on top of a graphene-iridium moiré superstructure were investigated by Fourier transformation reflection absorption infrared spectroscopy under ultrahigh vacuum conditions at $\sim 120 \mathrm{~K}$. The infrared bands of $\mathrm{D}_{2} \mathrm{O}$ clusters differ significantly from those observed for bulk $\mathrm{D}_{2} \mathrm{O}$ amorphous solid water or crystalline ice phases. Blue shifted symmetric and asymmetric stretching bands with narrower band widths and modified band intensity ratios were observed, pointing to an enhanced internal order and a reduced nearest neighbor distance. Furthermore, two IR bands of 'dangling' deuterium atoms were detected originating from threefold coordinated water molecules at the surface of the clusters and at their interface to the graphene layer. The latter arose only with the transition from the water clusters to an amorphous solid water layer. We propose that upon coalescence, opposing local dipoles trigger a hydrogen bond rearrangement


at the interface. Our results represent a first step towards an atomistic understanding of water in confinement.

\section{Introduction}

Water is the most fundamental substance for life, yet the understanding of the principal properties of water in its liquid and solid form, especially in nanometer confined geometries, is far from being complete. The understanding of the molecular structure and dynamics of bulk water and water in confinement is of fundamental importance for solvation science and biological as well as geological processes. ${ }^{1-4}$ The motivation to study water in confinement is to gain a deeper understanding of the complex phase transitions of water and its local order 5 by minimizing the amount of involved molecules, and furthermore to investigate interactions with the confining environment. Recent scientific work concerns water at various interfaces, where it forms patterns of molecular wetting layers. It was reported that water on surfaces can form half-dissociated adlayers, ${ }^{6}$ ordered structures as $2 \mathrm{D}$ stripes and patches ${ }^{7}$ or selfassembled nano sized clusters. ${ }^{8}$ The interaction with a substrate is reported to lead to a preferential orientation of the water molecules, ${ }^{9-13}$ which hence has impact on micro- to macroscopic properties of water, e.g. the flux through channels inside filtration membranes ${ }^{14}$ or other nano channels. ${ }^{15}$ As the surface-to-volume ratio is high for nanomaterials, a better understanding of the interplay between enhanced surface tension and hydrogen bonding properties inside water nanodroplets and nanopores is highly desirable. ${ }^{16}$

It was recently demonstrated that frozen water clusters of $\sim 1 \mathrm{~nm}$ size can be periodically arranged on graphene/ $\operatorname{Ir}(111) .{ }^{8}$ The arrangement of the water molecules inside the droplets is presumed to be amorphous, but their internal structure and phase has not been experimentally determined to this point. The moiré pattern of graphene on $\operatorname{Ir}(111)$ has a periodicity of $2.53 \mathrm{~nm}$ and provides an elegant way to decorate the surface with a high density of small nanoparticles with diameters up to $1.5 \mathrm{~nm} .{ }^{17,18}$ At the so-called hexagonal closed packing 
(HCP) sites, the graphene layer has the smallest distance to the Ir substrate and acts as a preferred location for the accumulation of adatoms and adsorbed molecules into clusters via interaction with the substrate. ${ }^{18}$ At higher coverages, the water clusters coalesce, and are supposed to form a layer of amorphous solid water (ASW). ${ }^{8}$ It was found that at $100 \mathrm{~K}$ an ASW film forms on a silica film grown on Mo(112) single crystal, while an ice monolayer film forms on the surface at 140-150 K. ${ }^{19}$ Previously we investigated the atomic structure of Pt and Ir nanoclusters on a graphene/Ir(111) support. ${ }^{20,21}$ As a key result, we found that the nanoclusters undergo a compression of up to $4.8 \%$ in the Pt case, triggered by the interaction with the graphene and the reduced geometry. The lattice compression can be translated to an effective pressure of $\sim 100 \mathrm{MPa}$ which, if also acting on water clusters, may result in interesting phase changes according to the water phase diagram. Such changes in the inter or intra molecular distances may have strong impact on water in solvation processes. Further on, density functional theory calculations for water molecules adsorbed on flat graphene supported by a $\mathrm{Cu}(111)$ surface as a weakly interacting metal (comparable to Ir (111)) resulted in slightly lower binding energies for water molecules with oxygen pointing downward to the graphene layer. ${ }^{9}$ For the water cluster phase, the same question of molecular water orientation at the interface arises.

To investigate the molecular water $\mathrm{D}_{2} \mathrm{O}$ arrangement of the graphene/Ir(111) supported nanoclusters and their vibrational dynamics we utilized high resolution infrared reflection absorption spectroscopy (IRRAS). IR spectroscopy is the method of choice since the absorption band frequencies as well as their widths are strongly dependent on the water phase, pressure, temperature and sample history. ${ }^{19,22-24}$ The overall shape of the spectral regime between $2300-2650 \mathrm{~cm}^{-1}$ serves as a fingerprint of specific phases and allows one to identify subtle structural changes, which is generally a challenge for nano-confined water. 


\section{Experimental}

All experiments are carried out at the DESY NanoLab. ${ }^{25}$ The $\operatorname{Ir}(111)$ single crystal was mounted on a molybdenum sample holder by tantalum clips and introduced into a preparation chamber $\left(p_{\text {base }}<10^{-10}\right.$ mbar $)$, which is part of an UHV transfer system. The sample was further prepared as depicted in Fig. 1 and described in the following. The crystal surface was cleaned by repeated Ar sputtering cycles (60 min, $p_{\mathrm{Ar}}=5 \cdot 10^{-6} \mathrm{mbar}$ ) and annealing in $\mathrm{O}_{2}\left(T=1300 \mathrm{~K}, p_{\mathrm{O}_{2}}=5 \cdot 10^{-7} \mathrm{mbar}\right)$ and vacuum $(T=1500 \mathrm{~K})$. After cooling to room temperature, the sample was exposed to $1 \cdot 10^{-7}$ mbar ethylene $\left(\mathrm{C}_{2} \mathrm{H}_{4}\right)$ for $45 \mathrm{~s}$. Annealing for $10 \mathrm{~min}$ at $1400 \mathrm{~K}$ results in the dehydrogenation of the ethylene and formation of small graphene islands on the surface. The flakes orient at the elevated temperature in a $\mathrm{R} 0^{\circ}$ rotation with respect to the $\operatorname{Ir}(111)$ facet. Further exposure of $\mathrm{C}_{2} \mathrm{H}_{4}$ at $1200 \mathrm{~K}$ results in the growth of the graphene islands until a complete monolayer covers the iridium surface. Subsequent flash annealing was carried out to heal defects in the graphene layer. Low energy electron diffraction and Auger electron spectrocopy were carried out to probe the generation of the $\mathrm{Gr} / \operatorname{Ir}(111)$ moiré structure (see Fig. S2 and S3). Shortly before the transfer, the samples are heated within the preparation chamber to at least $500 \mathrm{~K}$ at a base pressure better than $5 \cdot 10^{-11}$ mbar in order to remove captured adsorbates.

The infrared spectra were obtained by a FT-IRRAS, with capability to operate at ultrahigh vacuum conditions. The sample was transferred to the IR measurement chamber at a base pressure of $5 \cdot 10^{-10}$ mbar after cooling the sample station to $150 \mathrm{~K}$ by liquid $\mathrm{N}_{2}$ 


\section{Results and Discussion}

In the following, we will present the FT-IR results as a function of the $\mathrm{D}_{2} \mathrm{O}$ exposure. Details of the sample preparation and the experimental setup are summarized in the methodology section below and the supporting information (SI). Fig. 2 shows the IR spectra from the $\mathrm{Gr} / \mathrm{Ir}(111)$ surface at $117 \pm 3 \mathrm{~K}$ as a function of $\mathrm{D}_{2} \mathrm{O}$ exposure between 0-50 Langmuir (L). The IR bands in this work are mainly originating from the $\mathrm{D}_{2} \mathrm{O}$ molecules which are adsorbed perpendicular to the surface, as the detection of the flat-laying molecules on metal surfaces in IRRAS is difficult due to the so called surface selection rules. ${ }^{27}$ According to Ref. 8 , the ASW cluster phase is expected to form on the surface up to a coverage of about $2 \mathrm{~L}$ 

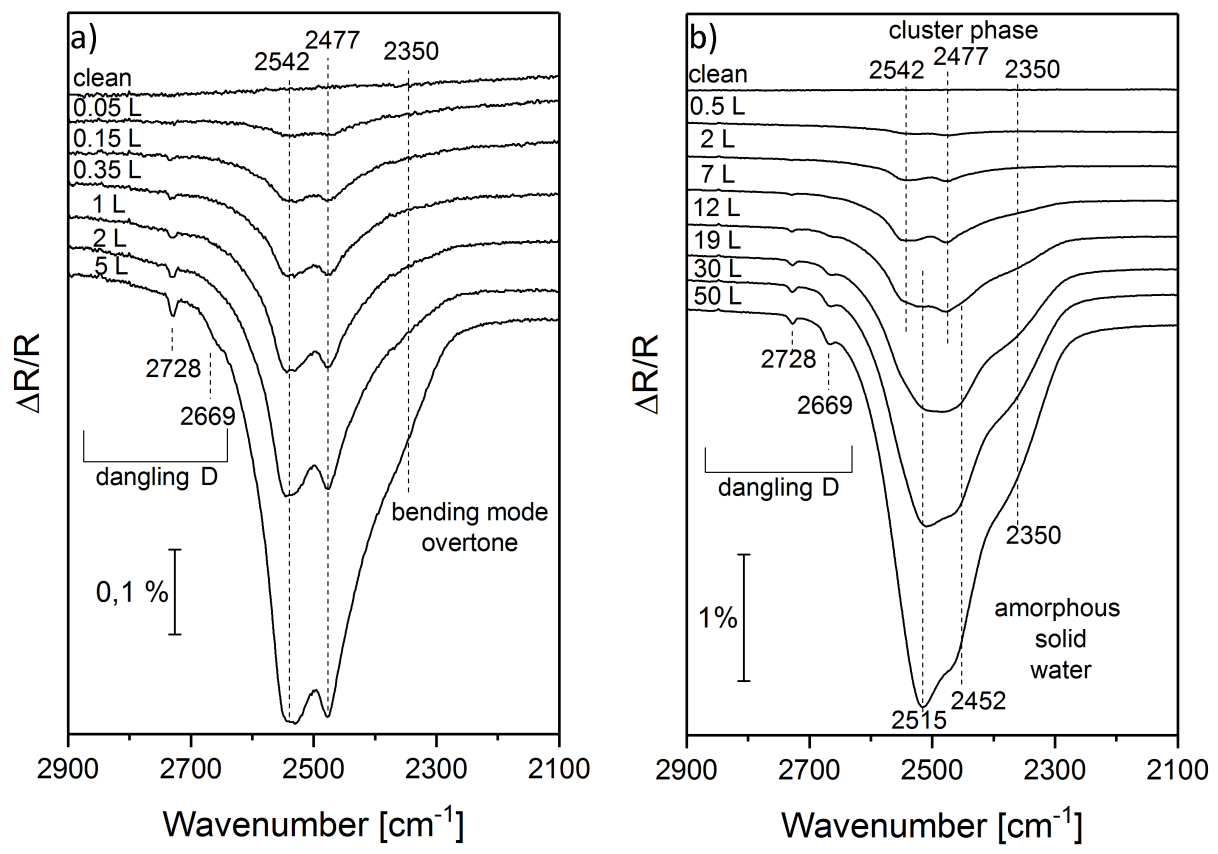

Figure 2: IR spectra in dependence of $\mathrm{D}_{2} \mathrm{O}$ exposure on the $\mathrm{Gr} / \mathrm{Ir}(111)$ substrate. a) Exposures up to $\sim 5 \mathrm{~L}$ result in stretching bands associated with the $\mathrm{D}_{2} \mathrm{O}$ ASW cluster phase. b) Exposures larger than $\sim 7 \mathrm{~L}$ result in stretching contributions at lower wavenumbers, which can be associated with the presence of an ASW film.

with $120 \mathrm{D}_{2} \mathrm{O}$ molecules per cluster. The spectra show the regime of the stretching bands $\left(\sim 2300-2650 \mathrm{~cm}^{-1}\right)$ and the bending mode overtone $\left(2350 \mathrm{~cm}^{-1}\right)$ as well as two features of dangling deuterium atoms $\left(2728 \mathrm{~cm}^{-1}, 2669 \mathrm{~cm}^{-1}\right)$, which we assign to under-coordinated water molecules. ${ }^{19,22,28-30}$ The dangling deuterium band at $2699 \mathrm{~cm}^{-1}$ was not reported in IR studies of the ASW on oxide and metal surfaces. Additionally, the bending mode fundamental and the association band are shown in Figure S1.

In Fig. 2a, the data for the exposure regime up to $5 \mathrm{~L}$ are plotted: the symmetric stretching band $\left(v_{\mathrm{s}}\right)$ of $\mathrm{D}_{2} \mathrm{O}$ is located at $2477 \mathrm{~cm}^{-1}$, whereas the $\mathrm{D}_{2} \mathrm{O}$ asymmetric stretching band $\left(v_{\text {as }}\right)$ is at $2542 \mathrm{~cm}^{-1}$ for this low coverage regime. The band positions are summarized in Table 1. These modes represent the most dominant vibrational bands in the spectrum. In Fig. 2b the data for an exposure up to $50 \mathrm{~L}$ are summarized. The rise of contributions at $2515 \mathrm{~cm}^{-1}$ and $2452 \mathrm{~cm}^{-1}$ are observed with increasing exposure above $5 \mathrm{~L}$, at which the water clusters were observed by scanning tunneling microscopy to coalesce. ${ }^{8}$ Their line 
shape and spectral position are in good agreement with bulk amorphous solid water (ASW film). ${ }^{23,24,28,29}$ Kaya et al. reported a long-range ordered structure of the ASW on the silica surface at $100 \mathrm{~K}$ by low energy electron diffraction showing a $\mathrm{c}(2 \times 2)$ superstructure with respect to the silica substrate. ${ }^{19}$ This observation was further verified by IR spectra showing a broad IR band centered at $3400 \mathrm{~cm}^{-1}$ originating from ASW. ${ }^{19}$ In comparison with the signal from the ASW layer, generated for exposures larger than $\sim 5-7 \mathrm{~L}$, the stretching modes of the ASW cluster phase forming at $\mathrm{D}_{2} \mathrm{O}$ exposures below $5 \mathrm{~L}$ are blue shifted by $\sim 25 \mathrm{~cm}^{-1}$. Also, a noticeable gap between the stretching contributions is present, which was not reported for ASW films, liquid water or crystalline ice so far.

The ASW cluster phase bands at $2542 \mathrm{~cm}^{-1}$ and $2477 \mathrm{~cm}^{-1}$ show narrower widths than the bands observed in bulk amorphous solid water spectra. The band width of water IR spectra is strongly dependent on the intermolecular distance distribution. An increased internal order reduces the distance distribution between the water molecules, decreasing the band width in IR spectra and thus explaining the gap between the two stretching modes. We can thus deduce that the ASW clusters show a higher degree of internal molecular order compared to ASW films, which may be attributed to the interaction with the graphene layer or the reduced number and nearest neighbors.

The $\sim 25 \mathrm{~cm}^{-1}$ blue shift of the ASW cluster stretching modes compared to the stretching modes of the amorphous solid water film can be explained by an enhancement of intramolecular bond strength between $\mathrm{O}$ and $\mathrm{D}$, as a result of the confined geometry. In contrast, the bending mode fundamental position at $1215 \mathrm{~cm}^{-1}$ was not changing over the whole exposure range up to $200 \mathrm{~L}$ (see Figure S1). This may be related to the fact that the water molecule bond angle does not change from the ASW cluster phase to the ASW film phase.

The development of the bending mode overtone sets in at exposures of 2-5 L, pointing to a reduction of the Fermi resonance between the symmetric stretch band and the bending mode overtone for the ASW cluster phase. An explanation for this observation can be found in the blue shift of the stretch bands. A Fermi resonance leads to a coupling between the 
symmetric stretch mode and bending mode overtone which is possible because of nearly identical vibration frequencies and the same Mulliken symmetry. As a result of the coupling an enhancement of the bending mode overtone band together with a decrease in stretching vibration band intensity is expected, together with an enhanced splitting of the involved bands. ${ }^{31,32}$

For the ASW cluster phase for exposures up to $5 \mathrm{~L}$ the intensity of the symmetric stretch mode at $2477 \mathrm{~cm}^{-1}$ is nearly identical to that one of the asymmetric stretch band at $2542 \mathrm{~cm}^{-1}$. The symmetric stretch band was observed to get depopulated relative to the asymmetric stretch band with increasing exposure during cluster coalescence in conjunction with the ASW film formation, see Fig. 2b. At the same time, the bending mode overtone at $2350 \mathrm{~cm}^{-1}$ was observed to increase in intensity, supporting the hypothesis of an enhanced mode coupling for the ASW film phase at higher exposures.

In the obtained spectra, a small band was observed for all exposures at $2728 \mathrm{~cm}^{-1}$. The position and intensity of this band suggests that it originates from threefold coordinated water molecules (one hydrogen donor bond, two hydrogen acceptor bonds) directing one 'dangling deuterium atom' (dD) towards the vacuum. ${ }^{29,30,33}$ A feature corresponding to twofold coordinated water molecules is not present in the spectrum, as it is expected to appear at higher wavenumbers $\left(2748 \mathrm{~cm}^{-1}\right) .{ }^{33}$ Since the band is present for all exposures, it is attributed to $\mathrm{dD}$ bonds at the nanocluster and ASW film surface.

Next we will discuss an atomic scale scenario for the ASW cluster and film formation based on the interpretation of our IR results (see Fig. 3): 1. at low exposures, water molecules adsorb on the surface and diffuse into minima of the potential landscape, i.e. the HCP sites (Fig. 3a) presumably with a preferred orientation facing the oxygen atom towards the graphene layer, because of the slight $p$ doping of graphene on $\operatorname{Ir}(111) .{ }^{34} 2$. Upon further exposure of $\mathrm{D}_{2} \mathrm{O}$, the molecules accumulate into clusters at the HCP sites of the moiré pattern as observed by STM. ${ }^{8}$ A strong interaction of water with FCC and HCP rather than TOP domains was observed in the STM images of monolayer water molecules on moiré -structured 


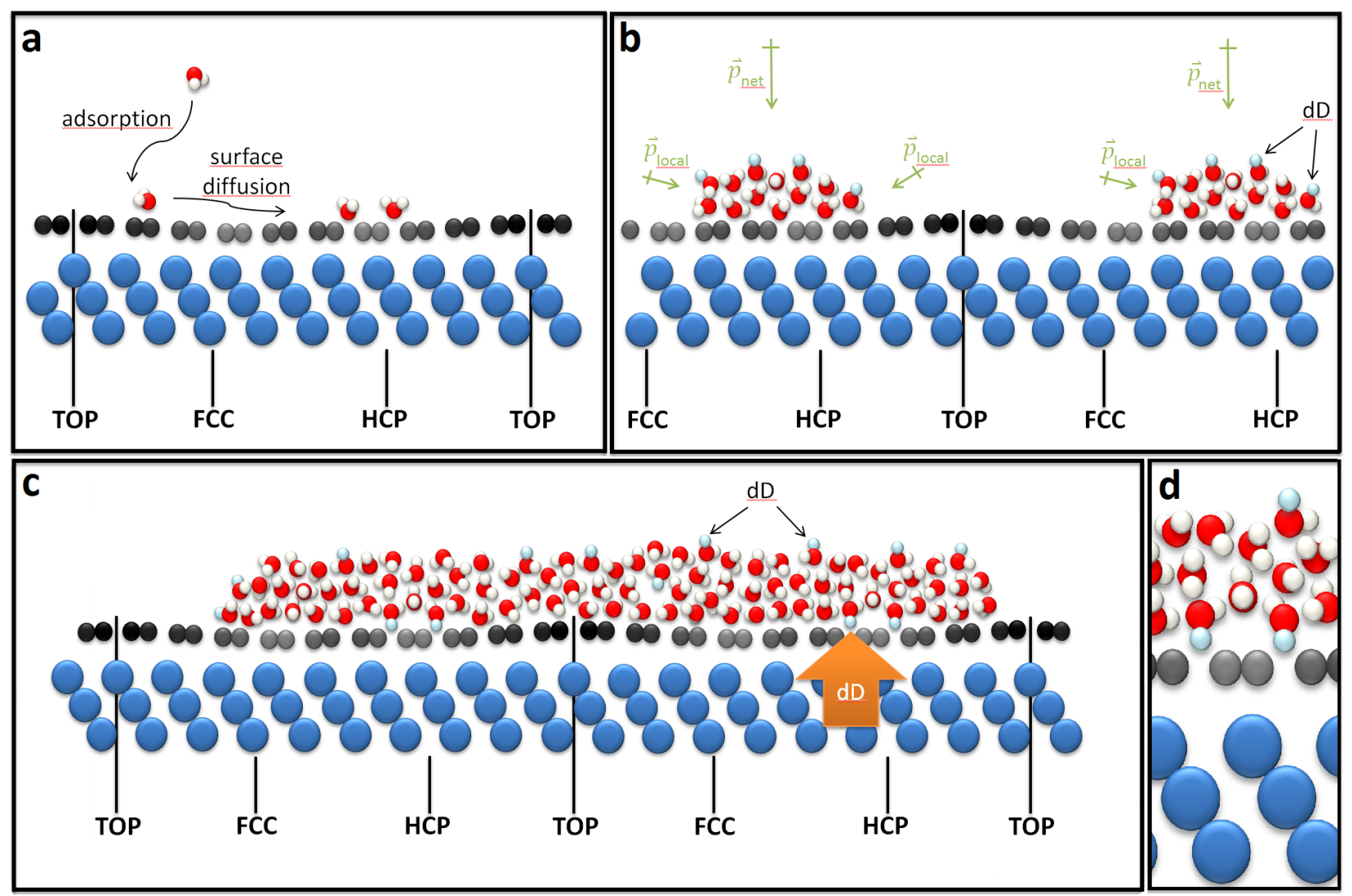

Figure 3: Proposed model of the molecular arrangement for different $\mathrm{D}_{2} \mathrm{O}$ exposure regimes. a) Molecular adsorption and surface diffusion of $\mathrm{D}_{2} \mathrm{O}$ molecules. b) Accumulation of molecules to water nanoclusters with an electrostatic net dipole and dangling deuterium atoms facing towards the vacuum. c) Amorphous solid water film after the coalescence of the clusters. Due to rearrangement, dangling deuterium atoms are facing towards the $\mathrm{Gr} / \operatorname{Ir}(111)$ support. The net dipole is zero or negligible at this point. d) Close view on the $\mathrm{dD}$ atoms at the $\mathrm{D}_{2} \mathrm{O}-\mathrm{Gr} / \operatorname{Ir}(111)$ interface. These features are not necessarily located at the HCP sites of the moiré pattern. 
FeO film on $\mathrm{Pt}(111)$ by Merte et al. ${ }^{35}$ The lower interaction of water with the TOP domain was attributed to the weaker electrostatic field above oxygen atoms at the TOP sites, resulting from the smaller rumpling of the FeO. ${ }^{35}$ At this point two contrary effects may occur: a) the molecules orient the oxygen side to the partially positive deuterium terminated side of the initial molecules; b) the tendency to generate hydrogen bonds to neighboring molecules orients the molecules individually in order to maximize their coordination number. At the surface of the clusters, threefold coordinated water molecules remain, directing dangling deuterium atoms towards the vacuum $\left(2728 \mathrm{~cm}^{-1}\right)$. As the available thermal energy is low, the orientations will not necessarily be thermodynamically favored. An electrical net dipole $\vec{p}$ remains in the clusters (Fig. 3b). The existence of a net dipole for small water clusters was reported by Su et al., ${ }^{36}$ investigating water clusters on $\mathrm{Pt}(111)$ surfaces at 120-137 K.

3. For further water exposure, the droplets grow in size. Just before coalescence sets in at $\sim 5 \mathrm{~L}$, the surfaces of neighboring nanoclusters are close and have local dipoles in opposing directions. Upon coalescence, a cascade of rearrangement by deuterium exchange sets in, in order to minimize the electrostatic repulsion and an amorphous solid water film is formed with a lower degree of internal order. Water molecules at the interface to the $\operatorname{Gr} / \operatorname{Ir}(111)$ support find no neighboring molecules to bond with, remaining undercoordinated (Fig. 3c and $3 \mathrm{~d}$ ), facing $\mathrm{dD}$ to the substrate which are affected by the proximity of the surface $\left(2669 \mathrm{~cm}^{-1}\right)$. Reorientation of hydrogen bonds was previously reported upon heating. ${ }^{37}$ Here the reorientation is proposed to be triggered by the opposing net dipoles of the clusters as coalescence takes place. The reorientation process and the formation of dangling D bonds at the interface is further supported by the occurrence of a new band at $2669 \mathrm{~cm}^{-1}$ (see Figure 2b), which only started to appear at higher exposures for which the amorphous solid water film forms.

Finally we discuss the annealing behavior of the ASW cluster and film phases, demonstrating the power of FT-IR spectroscopy in the identification of adsorbed water phases on surfaces. By repeated IR measurements while increasing the sample temperature from 

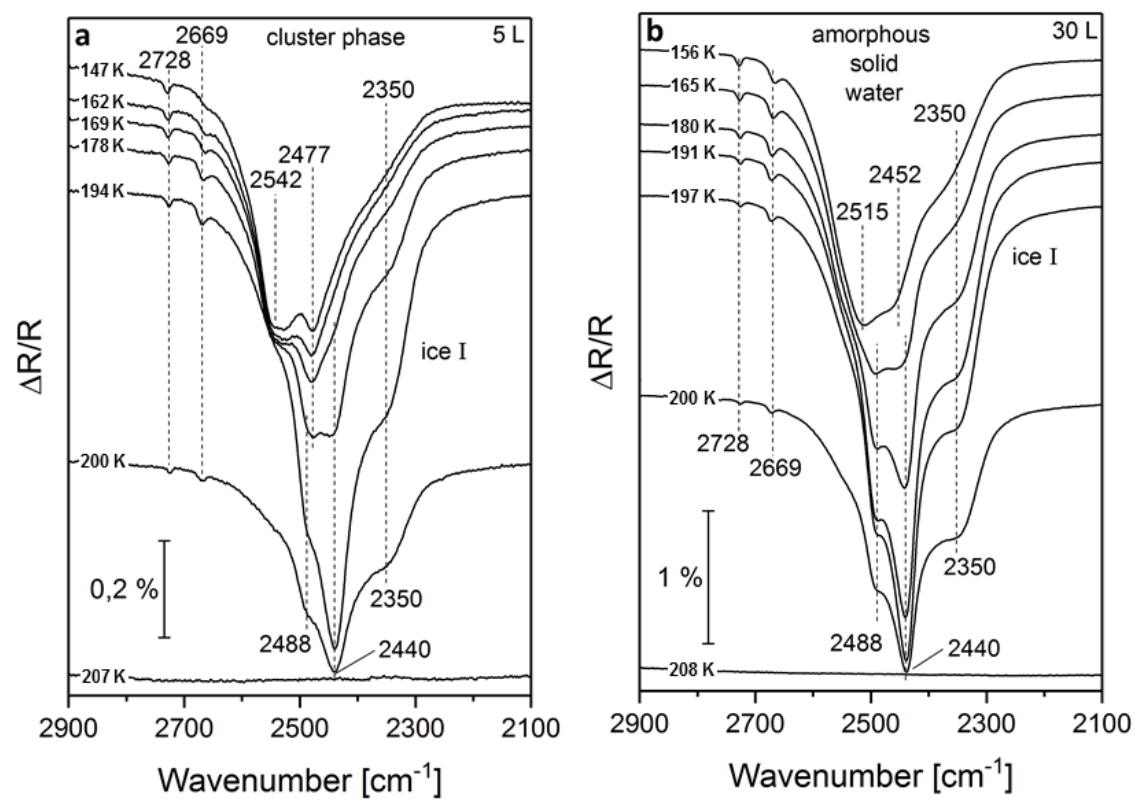

Figure 4: IR spectra of the annealing of $\mathrm{Gr} / \mathrm{Ir}(111)$ under UHV after a) dosing $5 \mathrm{~L}$ (ASW cluster phase) and b) $30 \mathrm{~L}$ (ASW film) of $\mathrm{D}_{2} \mathrm{O}$. The error bar of the temperatures given is $\pm 20 \mathrm{~K}$. 
With increasing temperature to about $194 \mathrm{~K}$, the spectrum transformed completely: the asymmetric and symmetric stretch bands of ice I were observed at $2488 \mathrm{~cm}^{-1}$ and $2440 \mathrm{~cm}^{-1}$, respectively, the latter being the most intense component in the spectrum due to the higher crystalline order of the bulk ice I phase. At higher temperatures, desorption started, which was complete at about $207 \mathrm{~K}$, inline with Ref. 8. At the same time, the bending mode overtone band at $2350 \mathrm{~cm}^{-1}$ got more enhanced, which is related to the enhanced Fermi resonance with the symmetric stretch band of ice I, now positioned at lower wave numbers (2440 $\mathrm{cm}^{-1}$ as compared to $2477 \mathrm{~cm}^{-1}$ for the ASW cluster phase). Overall, also the integrated IR signal increased, which can have the possible cause that after annealing the water molecules in the crystalline ice I phase arrange in such a way, that they exhibit a larger net dipole moment perpendicular to the surface. The two dangling $\mathrm{D}$ features at $2728 \mathrm{~cm}^{-1}$ and $2669 \mathrm{~cm}^{-1}$ interestingly were present after the annealing process. This indicates, that even while rearranging the molecular orientation upon the crystallization process, a certain amount of water molecules stayed under coordinated.

For comparison, the annealing behavior of an amorphous solid water film formed after adsorption of $30 \mathrm{~L} \mathrm{D}_{2} \mathrm{O}$ at $120 \mathrm{~K}$ is presented in Fig. 4b). The onset of structural changes occurred again at about $156 \mathrm{~K}$ with a redshift of the stretching band frequencies to the values of crystalline bulk ice I, and a stronger increase of the symmetric stretch band signal, indicative for an increased ordering. With increased annealing temperature, the symmetric stretch band at $2440 \mathrm{~cm}^{-1}$ increased more strongly in intensity, inline with the formation of crystalline ice I. The O-D dangling bond signals at $2728 \mathrm{~cm}^{-1}$ and $2669 \mathrm{~cm}^{-1}$ were observed throughout the entire temperature cycle, inline with the interpretation given above. The desorption set also in at a temperature of $208 \mathrm{~K}$. We can conclude that both the ASW cluster phase as well as the film phase exhibit a transition to crystalline ice I before desorption. No intermediate transition from the cluster phase to the layered ASW phase was identified during the annealing process. 
Table 1: Summary of the vibration modes of $\mathrm{D}_{2} \mathrm{O}$ observed by IRRAS. All values are given in $\mathrm{cm}^{-1}$

\begin{tabular}{ccccc} 
& $\begin{array}{c}\text { bending } \\
\text { fundamental }\end{array}$ & $\begin{array}{c}\text { bending } \\
\text { overtone }\end{array}$ & \multicolumn{2}{c}{$\begin{array}{c}\text { stretching } \\
\text { fundamentals }\end{array}$} \\
\hline \hline ASW clusters & 1215 & 2350 & 2477 & 2542 \\
ASW film & 1215 & 2350 & 2452 & 2515 \\
Ice I & 1212 & 2355 & 2440 & 2488 \\
\hline dD: & towards vacuum: 2728 & towards substrate: 2669
\end{tabular}

\section{Conclusion}

In conclusion, we find that the adsorption of up to $\sim 5 \mathrm{~L}$ of $\mathrm{D}_{2} \mathrm{O}$ on a $\mathrm{Gr} / \mathrm{Ir}(111)$ moiré structure at $117 \mathrm{~K}$ leads to the generation of a water phase with a unique infrared spectrum. This spectrum originates from water ASW nanoclusters self assembled on the Gr/Ir(111) superstructure. The stretching bands of the cluster phase exhibit a narrowing of the band width, in combination with an enhancement of the symmetric stretch band signal, as compared to the thin film ASW phase formed after higher exposures. Both point to an arrangement with a higher degree of molecular order compared to bulk amorphous solid water. Further on, we observed a blue shift of the stretching bands of the cluster phase relative to the ASW film phase which we attribute to a slightly enhanced intramolecular bond strength. Weak but sharp bands at $2728 \mathrm{~cm}^{-1}$ are assigned to dangling deuterium atoms of threefold coordinated water molecules at the surface of the clusters, facing towards the vacuum. For exposures larger than $\sim 5 \mathrm{~L}$ the water nanoclusters coalesce into a layer of ASW, evidenced by the corresponding IR fingerprint. Triggered by the opposing local net dipoles of the clusters, a rearrangement process of the hydrogen bond network takes place. As a result, the dangling $\mathrm{D}$ atoms present at the water-graphene interface, giving rise to a second, red shifted line at $2669 \mathrm{~cm}^{-1}$. Furthermore, the IR results demonstrate that annealing of the cluster phase resulted in a transition to crystalline ice and an intermediate transition to thin film ASW was not observed. Our results show that the effect of net dipoles, as well as temperature dependent reorientation of the hydrogen bonds should be considered in order to understand 
phase transitions of water when interfaces are involved. In addition, they give evidence for a confinement induced compression of the ASW cluster phase, which is relevant for solvation and melting dynamics of water in reduced dimensions, as it occurs in biological or geological systems. Our experiments may trigger further experimental and theoretical studies to determine the atomic structure and dynamics of water in graphene supported nanoclusters.

\section{Conflicts of interest}

There are no conflicts to declare.

\section{Acknowledgement}

The authors thank Thomas Michely for fruitful discussions.

\section{Supporting Information Available}

\section{References}

(1) Ebbinghaus, S.; Kim, S. J.; Heyden, M.; Yu, X.; Heugen, U.; Gruebele, M.; Leitner, D. M.; Havenith, M. An extended dynamical hydration shell around proteins. Proceedings of the National Academy of Sciences of the United States of America 2007, 104, 20749-20752.

(2) Wernet, P.; Nordlund, D.; Bergmann, U.; Cavalleri, M.; Odelius, M.; Ogasawara, H.; Näslund, L. A.; Hirsch, T. K.; Ojamäe, L.; Glatzel, P.; Pettersson, L. G. M.; Nilsson, A. The Structure of the First Coordination Shell in Liquid Water. Science 2004, 304, 995999.

(3) Bjärneholm, O.; Hansen, M. H.; Hodgson, A.; Liu, L.-M.; Limmer, D. T.; 
Michaelides, A.; Pedevilla, P.; Rossmeisl, J.; Shen, H.; Tocci, G.; Tyrode, E.; Walz, M.M.; Werner, J.; Bluhm, H. Water at Interfaces. Chemical Reviews 2016, 116, 76987726, PMID: 27232062.

(4) Levinger, N. E. Water in Confinement. Science 2002, 298, 1722-1723.

(5) Perakis, F.; Amann-Winkel, K.; Lehmkühler, F.; Sprung, M.; Mariedahl, D.; Sellberg, J. A.; Pathak, H.; Späh, A.; Cavalca, F.; Schlesinger, D. Diffusive dynamics during the high-to-low density transition in amorphous ice. Proceedings of the National Academy of Sciences 2017, 114, 8193-8198.

(6) Feibelman, P. J. Partial dissociation of water on Ru (0001). Science 2002, 295, 99-102.

(7) Cerda, J.; Michaelides, A.; Bocquet, M.-L.; Feibelman, P. J.; Mitsui, T.; Rose, M.; Fomin, E.; Salmeron, M. Novel water overlayer growth on Pd (111) characterized with scanning tunneling microscopy and density functional theory. Physical Review Letters 2004, 93, 116101.

(8) Standop, S.; Michely, T.; Busse, C. $\mathrm{H}_{2} \mathrm{O}$ on Graphene/Ir(111): A Periodic Array of Frozen Droplets. The Journal of Physical Chemistry C 2015, 119, 1418-1423.

(9) Li, X.; Feng, J.; Wang, E.; Meng, S.; Klimes, J.; Michaelides, A. Influence of water on the electronic structure of metal-supported graphene: Insights from van der Waals density functional theory. Physical Review B 2012, 85, 085425.

(10) Ma, J.; Michaelides, A.; Alfe, D.; Schimka, L.; Kresse, G.; Wang, E. Adsorption and diffusion of water on graphene from first principles. Physical Review B 2011, 84, 033402.

(11) Leenaerts, O.; Partoens, B.; Peeters, F. Water on graphene: Hydrophobicity and dipole moment using density functional theory. Physical Review B 2009, 79, 235440.

(12) Hamada, I. Adsorption of water on graphene: A van der Waals density functional study. Physical Review B 2012, 86, 195436. 
(13) Ho, T. A.; Striolo, A. Polarizability effects in molecular dynamics simulations of the graphene-water interface. The Journal of Chemical Physics 2013, 138, 054117.

(14) Van der Bruggen, B.; Schaep, J.; Wilms, D.; Vandecasteele, C. Influence of molecular size, polarity and charge on the retention of organic molecules by nanofiltration. Journal of Membrane Science 1999, 156, 29-41.

(15) Fernandez-Cuesta, I.; Laura Palmarelli, A.; Liang, X.; Zhang, J.; Dhuey, S.; Olynick, D.; Cabrini, S. Fabrication of fluidic devices with $30 \mathrm{~nm}$ nanochannels by direct imprinting. Journal of Vacuum Science \& Technology B, Nanotechnology and Microelectronics: Materials, Processing, Measurement, and Phenomena 2011, 29, 06F801.

(16) Tomo, Y.; Askounis, A.; Ikuta, T.; Takata, Y.; Sefiane, K.; Takahashi, K. Superstable Ultrathin Water Film Confined in a Hydrophilized Carbon Nanotube. Nano Letters 2018, 18, 1869-1874, PMID: 29424547.

(17) Coraux, J.; N Diaye, A. T.; Busse, C.; Michely, T. Structural coherency of graphene on Ir (111). Nano Letters 2008, 8, 565-570.

(18) N’Diaye, A. T.; Gerber, T.; Busse, C.; Mysliveček, J.; Coraux, J.; Michely, T. A versatile fabrication method for cluster superlattices. New Journal of Physics 2009, 11, 103045.

(19) Kaya, S.; Weissenrieder, J.; Stacchiola, D.; Shaikhutdinov, S.; Freund, H.-J. Formation of an ordered ice layer on a thin silica film. The Journal of Physical Chemistry C 2007, $111,759-764$.

(20) Franz, D.; Runte, S.; Busse, C.; Schumacher, S.; Gerber, T.; Michely, T.; Mantilla, M.; Kilic, V.; Zegenhagen, J.; Stierle, A. Atomic structure and crystalline order of graphenesupported Ir nanoparticle lattices. Physical review letters 2013, 110, 065503.

(21) Franz, D.; Blanc, N.; Coraux, J.; Renaud, G.; Runte, S.; Gerber, T.; Busse, C.; Michely, T.; Feibelman, P. J.; Hejral, U. Atomic structure of Pt nanoclusters supported 
by graphene/Ir (111) and reversible transformation under CO exposure. Physical Review B 2016, 93, 045426.

(22) Wong, A.; Shi, L.; Auchettl, R.; McNaughton, D.; Appadoo, D. R. T.; Robertson, E. G. Heavy snow: IR spectroscopy of isotope mixed crystalline water ice. Physical Chemistry Chemical Physics 2016, 18, 4978-4993.

(23) Backus, E. H.; Grecea, M. L.; Kleyn, A. W.; Bonn, M. Surface crystallization of amorphous solid water. Physical Review Letters 2004, 92, 236101.

(24) Bensebaa, F.; Ellis, T. Water at surfaces: What can we learn from vibrational spectroscopy? Progress in Surface Science 1995, 50, 173-185.

(25) Stierle, A.; Keller, T. F.; Noei, H.; Vonk, V.; Roehlsberger, R. DESY NanoLab. Journal of Large-Scale Research Facilities JLSRF 2016, 2, 76.

(26) Noei, H.; Qiu, H.; Wang, Y.; Löffler, E.; Wöll, C.; Muhler, M. The identification of hydroxyl groups on ZnO nanoparticles by infrared spectroscopy. Physical Chemistry Chemical Physics 2008, 10, 7092-7097.

(27) Noei, H.; Jin, L.; Qiu, H.; Xu, M.; Gao, Y.; Zhao, J.; Kauer, M.; Wöll, C.; Muhler, M.; Wang, Y. Vibrational spectroscopic studies on pure and metal-covered metal oxide surfaces. physica status solidi (b) 2013, 250, 1204-1221.

(28) Bolina, A. S.; Wolff, A. J.; Brown, W. A. Reflection Absorption Infrared Spectroscopy and Temperature-Programmed Desorption Studies of the Adsorption and Desorption of Amorphous and Crystalline Water on a Graphite Surface. The Journal of Physical Chemistry B 2005, 109, 16836-16845.

(29) Engquist, I.; Lundström, I.; Liedberg, B.; Parikh, A. N.; Allara, D. L. Infrared characterization of amorphous and polycrystalline $\mathrm{D}_{2} \mathrm{O}$ ice on controlled wettability self- 
assembled alkanethiolate monolayers. The Journal of Chemical Physics 1997, 106, $3038-3048$.

(30) Horn, A. B.; Chesters, M. A.; McCoustra, M. R.; Sodeau, J. R. Adsorption of stratospherically important molecules on thin $\mathrm{D}_{2} \mathrm{O}$ ice films using reflection absorption infrared spectroscopy. Journal of the Chemical Society, Faraday Transactions 1992, 88, $1077-1078$.

(31) De Marco, L.; Carpenter, W.; Liu, H.; Biswas, R.; Bowman, J. M.; Tokmakoff, A. Differences in the Vibrational Dynamics of $\mathrm{H}_{2} \mathrm{O}$ and $\mathrm{D}_{2} \mathrm{O}$ : Observation of Symmetric and Antisymmetric Stretching Vibrations in Heavy Water. The Journal of Physical Chemistry Letters 2016, \%, 1769-1774.

(32) de Marco, L.; Ramasesha, K.; Tokmakoff, A. Experimental Evidence of Fermi Resonances in isotopically dilute water from ultrafast broadband IR spectroscopy. Jornal of Physical Chemistry B 2013, 117, 15319-15327.

(33) Rowland, B.; Devlin, J. P. Spectra of dangling OH groups at ice cluster surfaces and within pores of amorphous ice. The Journal of Chemical Physics 1991, 94, 812-813.

(34) Busse, C.; Lazić, P.; Djemour, R.; Coraux, J.; Gerber, T.; Atodiresei, N.; Caciuc, V.; Brako, R.; N’Diaye, A. T.; Blügel, S.; Zegenhagen, J.; Michely, T. Graphene on Ir(111): Physisorption with Chemical Modulation. Physical Review Letters 2011, 10\%, 036101.

(35) Merte, L. R.; Bechstein, R.; Peng, G.; Rieboldt, F.; Farberow, C. A.; Zeuthen, H.; Knudsen, J.; Lægsgaard, E.; Wendt, S.; Mavrikakis, M. Water clustering on nanostructured iron oxide films. Nature communications 2014, 5, 4193.

(36) Su, X.; Lianos, L.; Shen, Y. R.; Somorjai, G. A. Surface-Induced Ferroelectric Ice on Pt(111). Physical Review Letters 1998, 80, 1533-1536. 
(37) Shephard, J.; Salzmann, C. Molecular reorientation dynamics govern the glass transitions of the amorphous ices. The Journal of Physical Chemistry Letters 2016, 7, $2281-2285$.

(38) Hornig, D.; White, H.; Reding, F. The infrared spectra of crystalline $\mathrm{H}_{2} \mathrm{O}, \mathrm{D}_{2} \mathrm{O}$ and HDO. Spectrochimica Acta 1958, 12, 338-349.
\end{abstract}




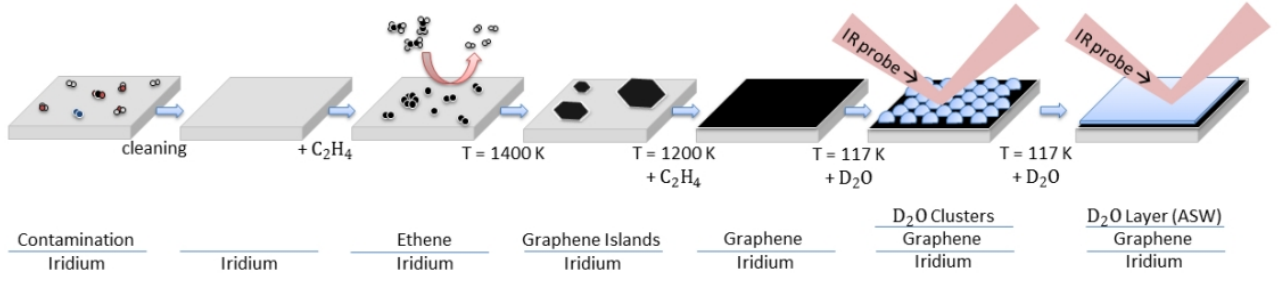

Figure 1: Sample preparation and D $\underline{2} \mathrm{O}$ dosing procedure. 

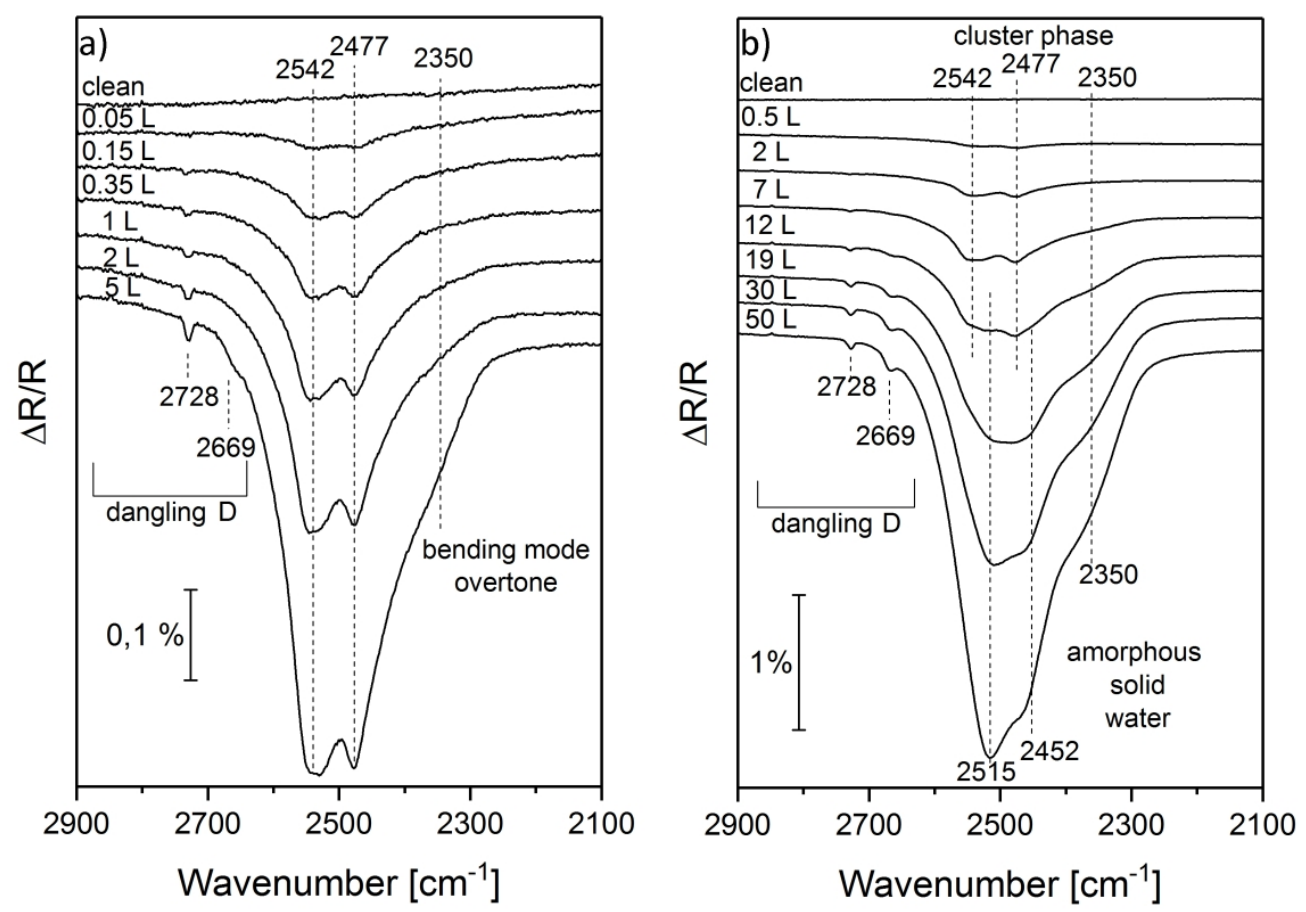

Figure 2: IR spectra in dependence of $\mathrm{D} 2 \mathrm{O}$ exposure on the $\mathrm{Gr} / \mathrm{Ir}(111)$ substrate. a) Exposures up to $\sim 5 \mathrm{~L}$ result in stretching bands associated with the D20 ASW cluster phase. b) Exposures larger than $\sim 7 \mathrm{~L}$ result in stretching contributions at lower wavenumbers, which can be associated with the presence of an ASW film. 

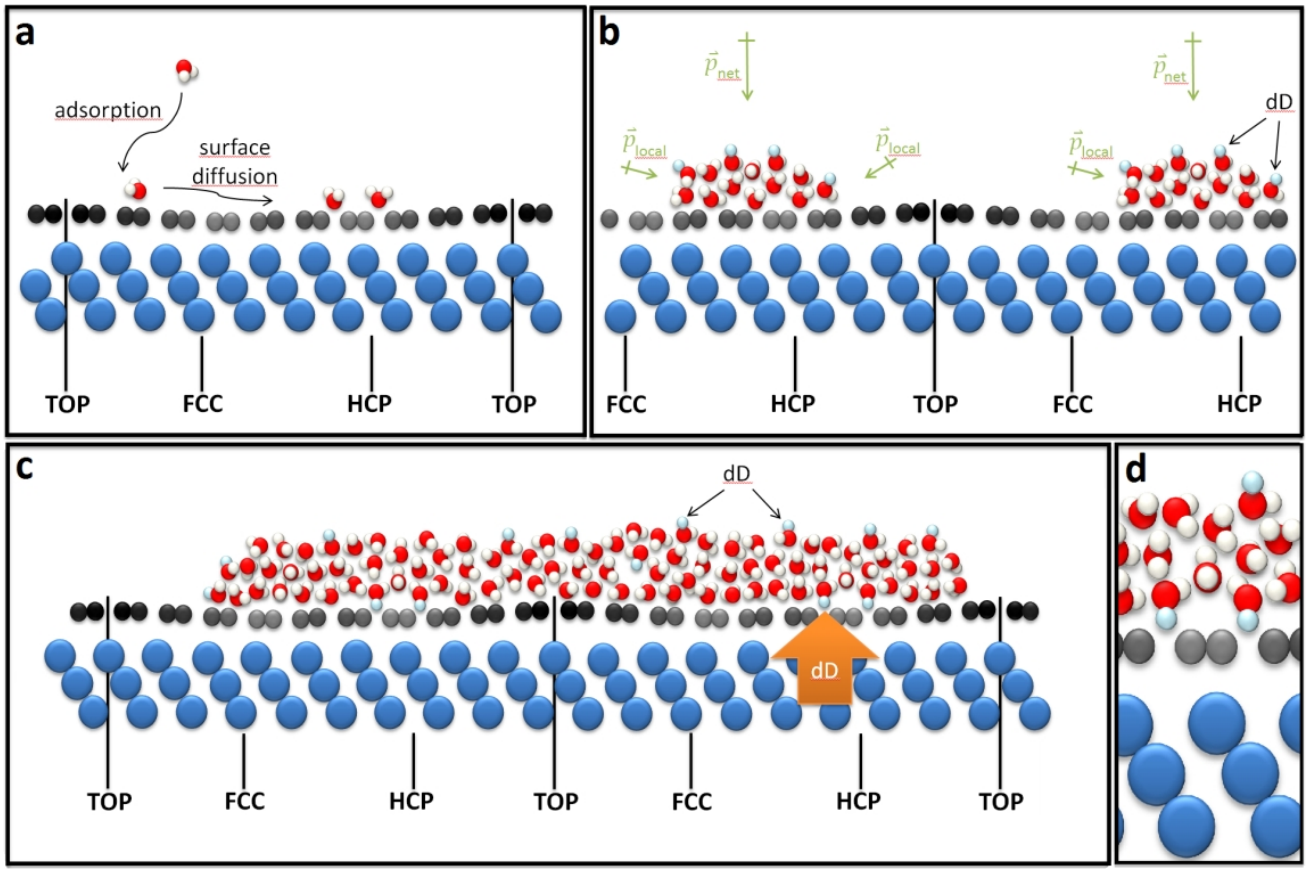

Figure 3: Proposed model of the molecular arrangement for different $D \underline{2} O$ exposure regimes. a) Molecular adsorption and surface diffusion of $\mathrm{D} 2 \mathbf{O}$ molecules. b) Accumulation of molecules to water nanoclusters with an electrostatic net dipole and dangling deuterium atoms facing towards the vacuum. c) Amorphous solid water film after the coalescence of the clusters. Due to rearrangement, dangling deuterium atoms are facing towards the $\mathrm{Gr} / \mathrm{Ir}(111)$ support. The net dipole is zero or negligible at this point. d) Close view on the dD atoms at the $\mathrm{D} \underline{\mathbf{2}} \mathrm{O}-\mathrm{Gr} / \mathrm{Ir}(111)$ interface. These features are not necessarily located at the HCP sites of the moiré pattern. 

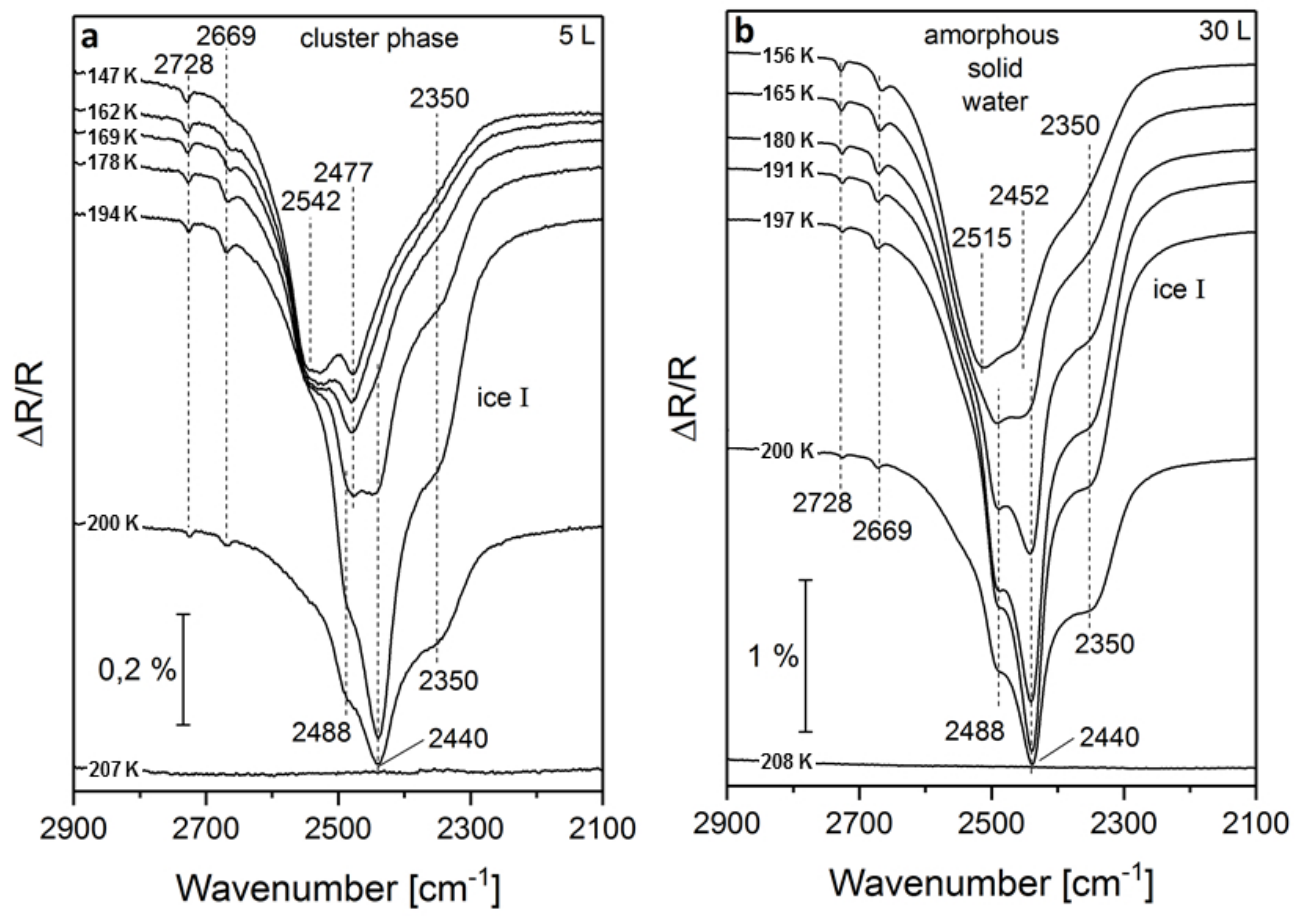

Figure 4: IR spectra of the annealing of $\mathrm{Gr} / \mathrm{Ir}(111)$ under UHV after a) dosing $5 \mathrm{~L}$ (ASW cluster phase) and b) $30 \mathrm{~L}$ (ASW film) of D2O. The error bar of the temperatures given is $\pm 20 \mathrm{~K}$. 


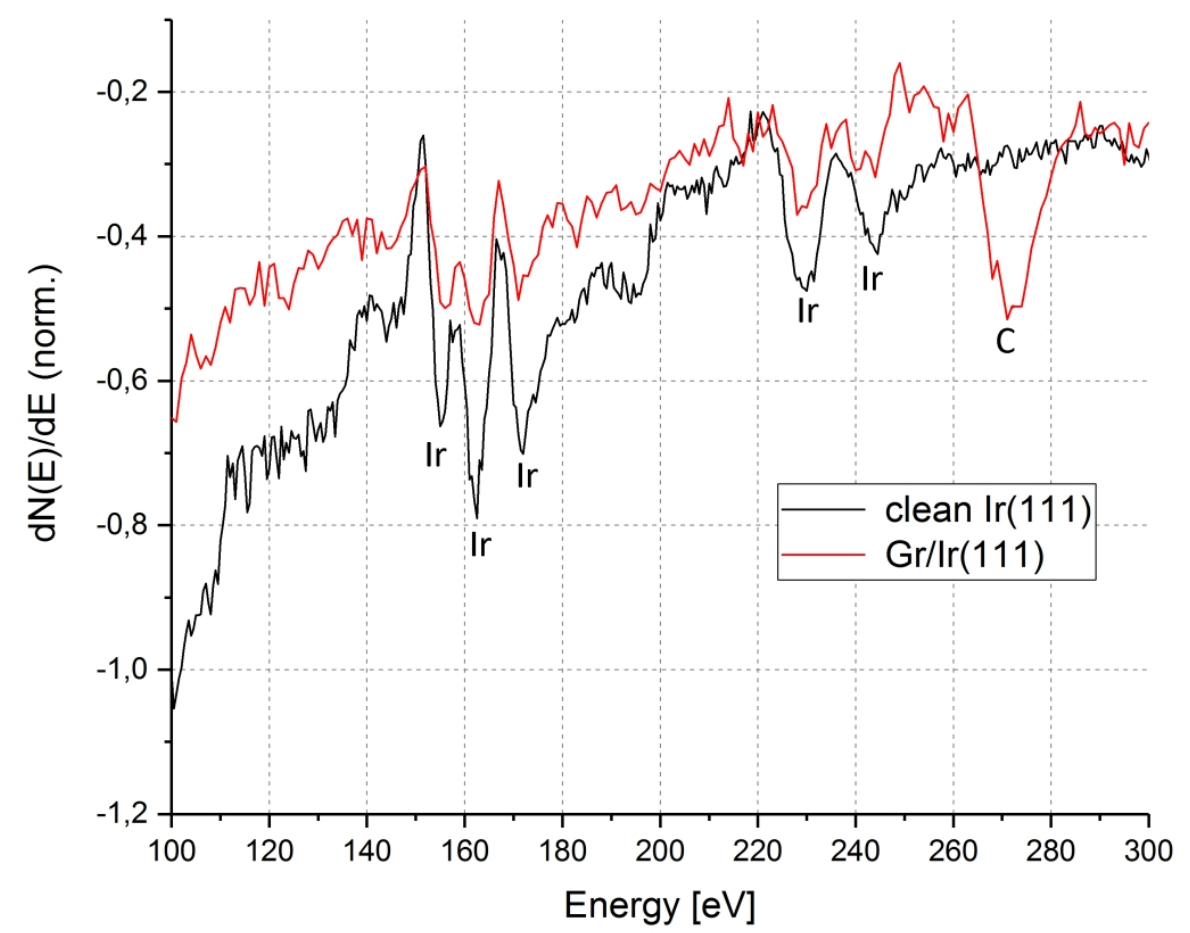

ACS Paragon Plus Environment 


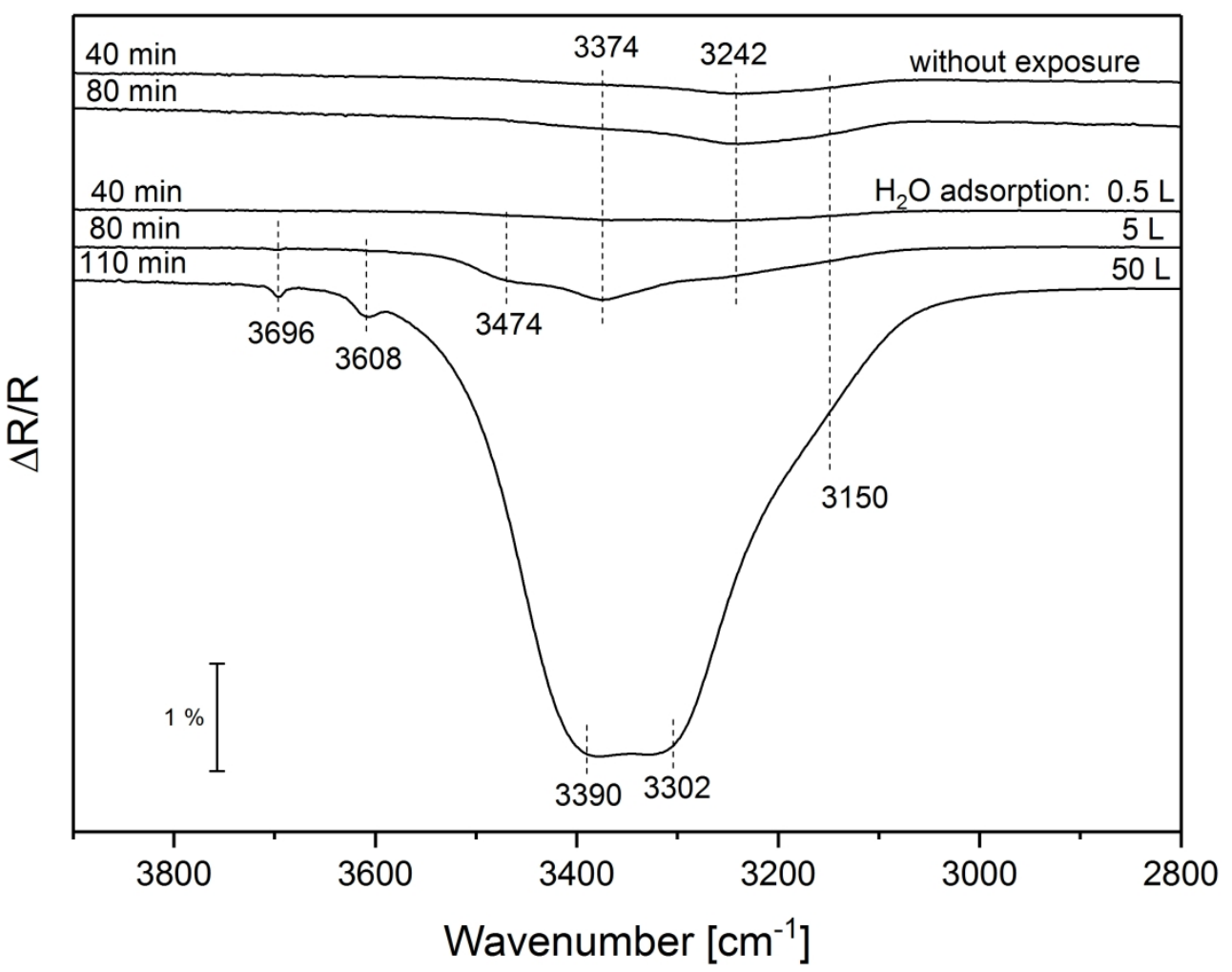

Figure 1: Exposure dependent $\mathrm{D} \underline{2} \mathrm{O}$ adsorption on $\mathrm{Gr} / \mathrm{Ir}(111)$ : association bands and bending mode fundamental 


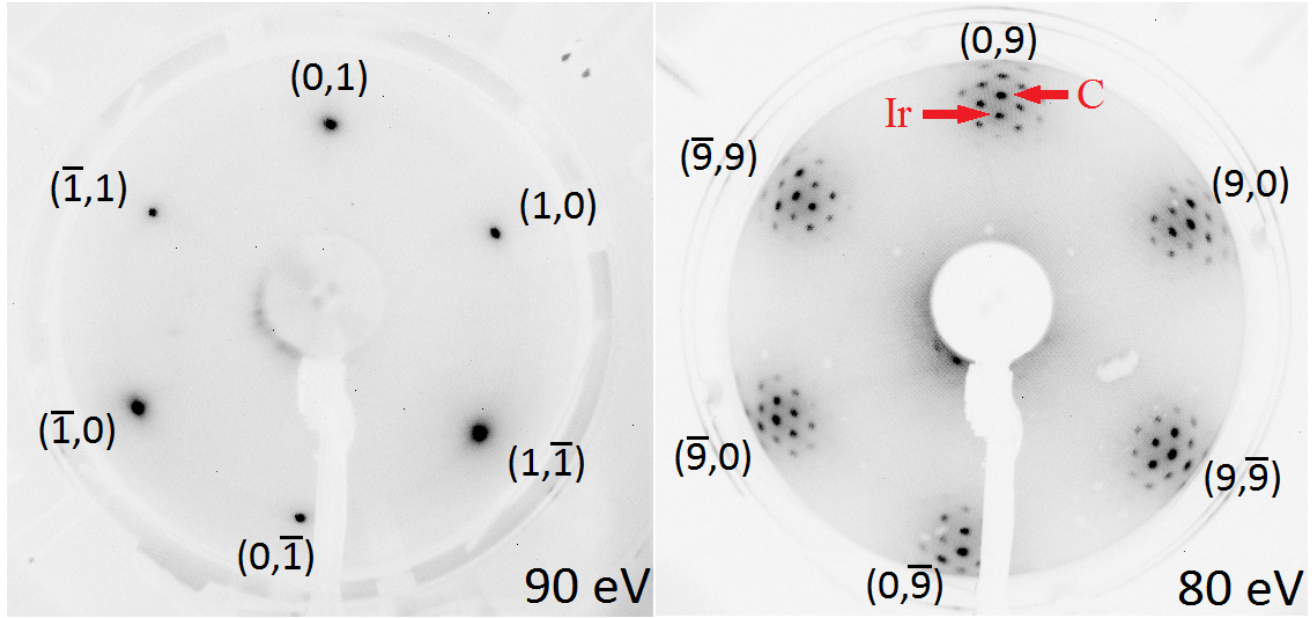

Figure 2: Low energy electron diffraction pattern in reversed contrast. In the center, the backside of the electron gun covers the (00) reflex. Left: Diffraction pattern of the cleaned $\operatorname{Ir}(111)$ surface. Right: Diffraction pattern of the Gr/Ir(111) moir'e structured surface. 
Figure 4: Schematic image of the FT-IRRAS. The interferometer and detector compartment are evacuated to 3 mbar, whereas the sample environment is under UHV conditions. It is possible to cool the manipulator and thus the sample with liquid nitrogen (LN2) flow to, and to heat the sample by filament heating. The lowest temperature obtained was $113 \mathrm{~K}$. Via the transfer rod, it is possible to pick up samples in the UHV transfer system and introduce them into the sample compartment. The IR source is a silicon carbide globar. $\mathrm{KBr}$ windows are separating the UHV chamber from the other compartments. 